\title{
Combinatorial Bayesian Dynamic Linear Models of Bridge Monitored Data and Reliability Prediction
}

\author{
Xueping Fan ${ }^{1,2}$ and Yuefei Liu ${ }^{1,2}$ \\ ${ }^{1}$ Key Laboratory of Mechanics on Disaster and Environment in Western China (Lanzhou University), \\ The Ministry of Education of China, China \\ ${ }^{2}$ School of Civil Engineering and Mechanics, Lanzhou University, Lanzhou 730000, China
}

Correspondence should be addressed to Xueping Fan; fanxp@lzu.edu.cn

Received 24 November 2015; Accepted 13 January 2016

Academic Editor: Ian Smith

Copyright ( $\odot 2016$ X. Fan and Y. Liu. This is an open access article distributed under the Creative Commons Attribution License, which permits unrestricted use, distribution, and reproduction in any medium, provided the original work is properly cited.

\begin{abstract}
Considering the uncertainties and randomness of the mass structural health monitored data, the objectives of this paper are to present (a) a procedure for effective incorporation of the monitored data for the reliability prediction of structural components or structures, (b) one transforming method of Bayesian dynamic linear models (BDLMs) based on 1-order polynomial function, (c) model monitoring mechanism used to look for possible abnormal data based on BDLMs, (d) combinatorial Bayesian dynamic linear models based on the multiple BDLMs and their corresponding weights of prediction precision, and (e) an effective way of taking advantage of combinatorial Bayesian dynamic linear models to incorporate the historical data and real-time data in structural timevariant reliability prediction. Finally, a numerical example is provided to illustrate the application and feasibility of the proposed procedures and concepts.
\end{abstract}

\section{Introduction}

Long-term ambient environments, such as chemical attack from environmental stressors and continuously increasing traffic volumes, make the physical quantities of civil infrastructure be subjected to changes in both time and space; these changes would make serious impacts on the serviceability and the ultimate capacity of structures and further have serious impacts on the remaining life of an existing structure [1]. The structural performances' degradation processes (e.g., resistance, reliability indices), which are usually considered as Markov chains, are time-variant and irreversible. The timevariant reliability indices of bridges are dependent on both the applied loads and the remaining strength of structural components or system, which can reflect the safety and serviceability of bridges, and the reliability indices can be solved with first-order second-moment (FOSM) method [2, 3]. Therefore, assessing as well as predicting the structural time-dependent reliability indices is crucial for structural safety and serviceability assessment.
Through health monitoring of bridges, the structural basic statuses, including strains, stresses, and deflections of specified structural components or structures, can be obtained. Nowadays the research on structural health monitoring (SHM) generally experiences two stages. The first stage, falling in the mature stage, is to install an array of sensors for the observation and collection of data on a bridge structure during a period of time [4-7]. The second stage is mainly the application of health monitoring information. A sound number of studies are mainly focused on the modal parameter identification, structural damage detection technology, performance prediction, reliability assessment, and other fields $[8,9]$. For research of the bridge reliability prediction and assessment, some achievements [10-14] are obtained, such as the reliability assessment of long span truss bridge, structural performance prediction based on monitored extreme data, and the use of the statistics of extremes to the reliability assessment and performance prediction of monitored highway bridges. However, due to the uncertainty of the bridges' real-time health monitored data, the research 
on real-timely predicting structural reliability is at the initial stage in the world.

In this paper, considering the uncertainty of mass monitored extreme data which is time-dependent monitored data in the past days, BDLMs are introduced to combine the monitored data with the structural reliability prediction. First, with the monitored data, the single BDLMs and the corresponding model monitoring mechanism are, respectively, given, then the combinatorial prediction model of monitored extreme data is firstly built based on the built single BDLMs and the corresponding weights of prediction precisions, and the prediction precisions between the combinatorial prediction model and the single BDLMs are compared. Finally the real-timely predicted reliability indices of bridge structures are obtained with FOSM method based on the proper prediction model of monitored data. The proposed models and procedures are applied to an existing bridge.

\section{Bayesian Dynamic Linear Models (BDLMs)}

BDLMs are the predicting approaches based on a philosophy of information updating $[15,16]$ which define a dynamic model system of time series processes that can incorporate all useful monitored information into the model to update the prediction model. The BDLMs include a state equation, an observation equation, the initial information, and the timedependent probability recursion processes based on Bayesian method. The state equation shows changes of the system with time and reflects inner dynamic changes of the system and random disturbances. The observation equation expresses the relationship between the measured data and the current state parameters of the system. According to the definition of BDLMs [15], for each time $t$, the general dynamic linear model is characterized by the quadruple $\left\{1, \alpha, V_{t}, W_{t}\right\}$ and formally defined as follows:

observation equation:

$$
y_{t}=\theta_{t}+v_{t}, \quad v_{t} \sim N\left[0, V_{t}\right], \quad(t=1,2, \ldots, T) ;
$$

state equation:

$$
\begin{aligned}
\theta_{t}=\alpha \theta_{t-1}+\zeta+\omega_{t}, & \\
& \omega_{t} \sim N\left[0, W_{t}\right],(t=1,2, \ldots, T) ;
\end{aligned}
$$

initial information:

$$
\left(\theta_{t-1} \mid \mathbf{D}_{t-1}\right) \sim N\left[m_{t-1}, C_{t-1}\right]
$$

where $y_{t}$ is the observation data at time $t$; $v_{t}$ is the observation error or the observation noise; $\theta_{t}$ is the state variable at time $t ; N[\cdot]$ is normal probability density function; $V_{t}$ is the variance which indicates the uncertainty of observation errors; $\alpha$ and $\zeta$ are both the regression coefficient of states; $W_{t}$ is the variance which indicates the model uncertainty recursive from time $t-1$ to time $t$. $\omega_{t}$ is the state error or state noise at time $t$; $T$ is the monitored total time; the initial information is the probabilistic representation of the predictors' belief about the level $\theta_{t-1}$ at time $t-1$. The mean value $m_{t-1}$ is a point estimate of this level $\theta_{t-1}$, and $C_{t-1}$ measures the associated uncertainty. Each information set $\mathbf{D}_{t-1}$ comprises all the information available at time $t-1$, including $\mathbf{D}_{0}$, the values of the variances $\left\{V_{t}, W_{t}: t>0\right\}$, and the values of the observations $y_{t-1}, y_{t-2}, \ldots, y_{1}$. Thus, the only new information becoming available at time $t$ is the observational value $y_{t}$, so $\mathbf{D}_{t}=\left\{y_{t}, \mathbf{D}_{t-1}\right\}$.

In this paper, the BDLMs mean that the observation equation and the state equation are both linear and are shown in (1) and (2). The 1-order polynomial function model is adopted to build the state equations.

\subsection{Transferred State Equation Based on 1-Order Polynomial} Function and Monitored Data. For the mass and random monitored extreme data, especially for monitored data at time $t-1$ and before time $t-1$, the discretized motion equation and the fitted 1-order polynomial function, which is commonly used for the prediction of the trend data, are adopted to predict future stress data of time $t$, so the 1-order polynomial function can be applied to properly build the BDLMs.

(1) 1-Order Polynomial Function of Monitored Data. Consider

$$
\theta_{t}=a+r t+e_{t} \quad(t=1,2, \ldots, T)
$$

where $\theta_{t}$ is the trend data (state variable) at time $t ; r, a$ are coefficients; $e_{t}$ is the state error indicating the model uncertainty; $T$ is the total monitored time, unit of which is day.

(2) State Equation Based on (4). The first-order differential of (4)

$$
d \theta_{t}=r d t+d e_{t} \quad(t=1,2, \ldots, T)
$$

was considered as the discretized motion equation, where $r$ is the nominal speed of the trend data $\theta_{t}$, which can be obtained with (4); $d e_{t}$ is an error term. For simplicity, we consider a discretization in small interval of time $(t-1, t)$, as follows:

$$
\frac{\theta_{t}-\theta_{t-1}}{t-(t-1)}=r+e_{t} \quad(t=1,2, \ldots, T)
$$

that is,

$$
\begin{aligned}
\theta_{t}=\theta_{t-1}+r\{t-(t-1)\}+e_{t}\{t-(t-1)\} & \\
& (t=1,2, \ldots, T),
\end{aligned}
$$

where it is assumed that the random error $e_{t}$ has density $N\left[0, W_{t}=\sigma_{e}^{2}\right]$ and $W_{t}$ can be estimated with (13). With a further simplification, we take unitary time intervals as one day; namely, $t-(t-1)=1$, so that (7) can be rewritten as follows:

$$
\theta_{t}=\theta_{t-1}+r+e_{t}, \quad(t=1,2, \ldots, T),
$$

where (8) will be used to build the state equation of BDLMs, which will be shown in (10). 


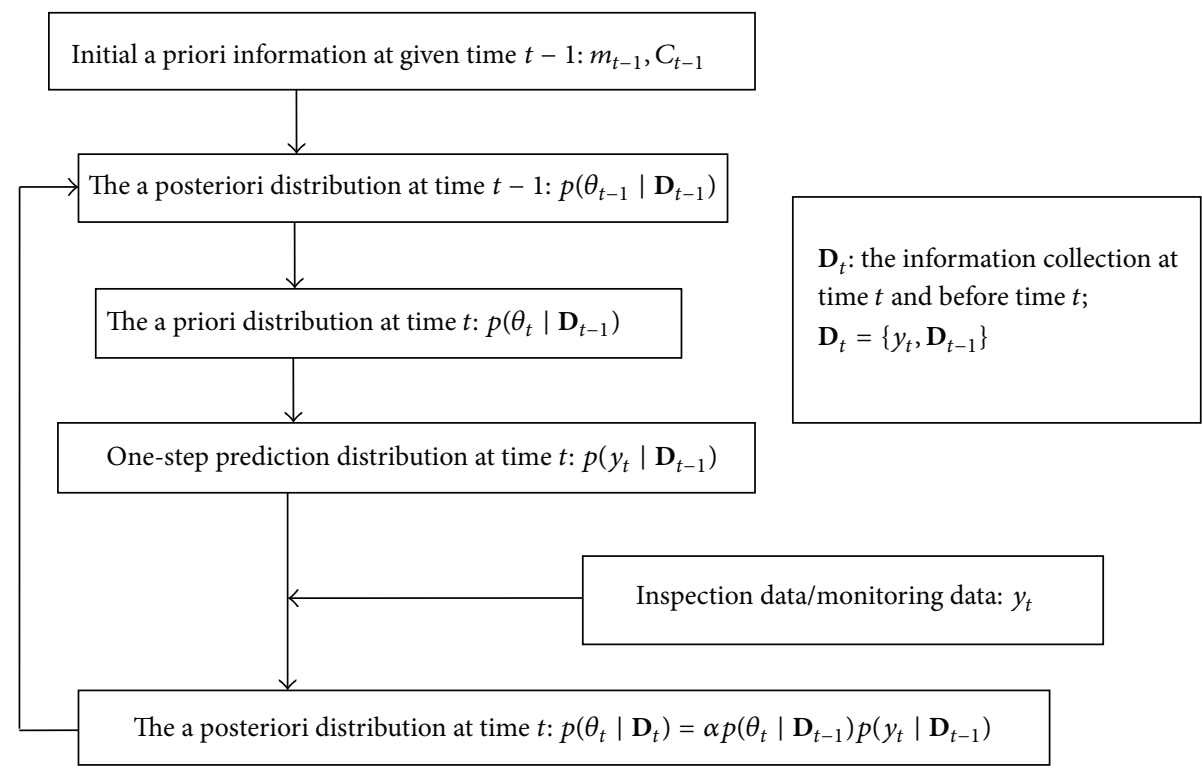

FIgURE 1: The modeling process of BDLMs.

2.2. Transferred BDLMs Based on 1-Order Polynomial Function. Based on Section 2.1, according to the definition of BDLMs $[15,17]$, for each time $t$, the general and easy forms of the dynamic linear models are defined as follows:

observation equation:

$$
y_{t}=\theta_{t}+v_{t}, \quad v_{t} \sim N\left[0, V_{t}\right], \quad(t=1,2, \ldots, T) ;
$$

state equation:

$$
\begin{aligned}
\theta_{t}=\theta_{t-1}+r+\omega_{t}, & \\
& \omega_{t} \sim N\left[0, W_{t}\right],(t=1,2, \ldots, T) ;
\end{aligned}
$$

initial information:

$$
\left(\theta_{t-1} \mid \mathbf{D}_{t-1}\right) \sim N\left[m_{t-1}, C_{t-1}\right] \text {, }
$$

where $y_{t}$ is the monitored data at time $t ; \theta_{t}$ is the state parameter indicating the level of the monitored data at time $t ; r$ is obtained with (4); $v_{t}$ and $\omega_{t}$ are, respectively, the monitored error and the state error at time $t$, which are all zero-mean normal random variables.

For each time $t$, the BDLMs include the following parameters: $V_{t}$ is the variance of monitored errors at time $t ; W_{t}$ is the variance of state error at time $t ; v_{t}$ and $\omega_{t}$ are, respectively, monitored errors and state errors. It is assumed that error sequences $v_{t}$ and $\omega_{t}$ are internally independent, mutually independent, and independent of $\left(\theta_{t-1} \mid \mathbf{D}_{t-1}\right)$.
With (9)-(11), the relationships between monitored data and state parameters are shown in

$$
\begin{aligned}
\left(y_{t} \mid \theta_{t}\right) & \sim N\left[\theta_{t}, V_{t}\right], \\
\left(\theta_{t} \mid \theta_{t-1}\right) & \sim N\left[\theta_{t-1}+r, W_{t}\right] .
\end{aligned}
$$

It can be known from (12) that the modeling processes of BDLMs can be divided into two key steps, which are shown in Figure 1. The first step is to obtain the a priori probability density function (PDF) of $\theta_{t}$ at time $t$ based on the state equation and the a posteriori PDF of $\theta_{t-1}$ at time $t-1$; the second step is to get the a posteriori PDF of $\theta_{t}$ at time $t$ based on the a priori PDF of state parameters at time $t$ and inspection/monitored data $y_{t}$ at time $t$.

In this paper, the monitored interval period of extreme stress data is one day; $V_{t}$ is estimated with the variance of monitored data. According to the research of [15], $W_{t}$ can be approximately solved with

$$
W_{t}=-G_{t} C_{t-1} G_{t}^{\prime}+\frac{C_{t-1}}{\delta}
$$

where $G_{t}^{\prime}$ is the transpose of $G_{t}$ and $\delta$ is the discount factor defined by engineering experience, which is usually $0.95-0.98$ for the BDLMs based on 1-order polynomial function.

\section{Prediction and Monitoring of Monitored Extreme Stress Based on Combinatorial BDLMs}

3.1. Assumptions of the BDLMs. BDLMs are presented as a special case of a general state-space model, being linear and Gaussian. So the BDLMs satisfy the assumptions of 
a state-space model. While the basic assumptions $[15,18]$ of state-space model are as follows:

(a) State variables, observation errors, and state errors all follow normal distributions. (b) $\left(\theta_{t}\right)$ is a Markov chain [15] which is shown as follows.

Dependence Structure for a State-Space Model. Consider

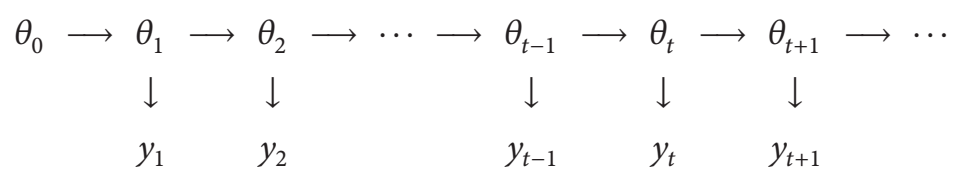

namely, $\pi\left(\theta_{t} \mid \mathbf{D}_{t-1}\right)=\pi\left(\theta_{t} \mid \theta_{0: t-1}, y_{1: t-1}\right)=\pi\left(\theta_{t} \mid\right.$ $\left.\theta_{t-1}\right)$, where $\pi(\cdot)$ is a general PDF.

(c) Conditionally on $\left(\theta_{t}\right)$, the $\left(y_{t}, t=1,2,3, \ldots, T\right)$ are independent of each other and $y_{t}$ depends on $\theta_{t}$ only (see (9)).

The recursive relation between state variables and inspection/monitored variables is shown in (14).

3.2. Combinatorial Prediction Model. Suppose that there are $n$ $(n=2,3, \ldots)$ BDLMs about monitored extreme data $[19,20]$; the $i$ th $(i=1,2, \ldots, n)$ dynamic linear models are as follows:

observation equation:

$$
\begin{aligned}
& y_{t}=\theta_{i, t}+v_{i, t}, \quad v_{i, t} \sim N\left[0, V_{i, t}\right],(t=1,2, \ldots, T) ; \\
& \text { state equation: } \\
& \theta_{i, t}=\theta_{i, t-1}+r_{i, t}+\omega_{i, t}, \\
& \qquad \omega_{i, t} \sim\left[0, W_{i, t}\right],(t=1,2, \ldots, T),
\end{aligned}
$$

where $r_{i, t}$ can be obtained with (4)-(8).

Normal a priori distribution about the initial information is as follows:

$$
\left(\theta_{i, t-1} \mid \mathbf{D}_{i, t-1}\right) \sim N\left[m_{i, t-1}, C_{i, t-1}\right],
$$

where $v_{i, t}$ is observational error of the $i$ th model; $\theta_{i, t}$ is state variable at time $t ; V_{i, t}$ is the variance of monitored errors; $W_{i, t}$ is the variance of state noise; $\omega_{i, t}$ is state error or state noise; $\mathbf{D}_{i, t}$ is the information set at time $t$ and before time $t$; and $\mathbf{D}_{i, t}=\left\{y_{t}, \mathbf{D}_{i, t-1}\right\} . \mathbf{D}_{i, t-1}$ is the information set at time $t-1$, including mean value $\left(m_{i, t-1}\right)$ and variance $\left(C_{i, t-1}\right)$. In addition, suppose that $v_{i, t}$ and $\omega_{i, t}$ are internally and mutually independent of each other, and they are independent of $\theta_{i, t}$.

If the initial state data follows the lognormal distribution, then the state data can be transformed into a quasinormal distribution $N\left[\mu^{\prime}, \sigma^{\prime 2}\right][21,22]$ with (18) and (19); the distribution parameters are, respectively, shown as

$$
\begin{aligned}
\sigma^{\prime} & =\frac{\phi(-1.645)}{g\left(x_{0}\right)}, \\
\mu^{\prime} & =x_{0}+1.645 \sigma^{\prime},
\end{aligned}
$$

where $g(\cdot)$ is the actual fitted PDF of the sample data (lognormal PDF), and the actual probability distribution function (lognormal probability distribution function) is $G(\cdot)$, and $G\left(x_{0}\right)=0.05$.

If the initial state data follows the other distributions, then the distribution can be approximately obtained as follows:

(1) With estimation method of kernel density, the actual distribution function $G\left(\theta_{t-1}\right)$ of the initial state data is approximately $g\left(\theta_{t-1}\right)$; namely,

$$
G\left(\theta_{t-1}\right) \approx g\left(\theta_{t-1}\right) .
$$

(2) Since any set of data can be fitted by a few normal distributions, namely,

$$
G\left(\theta_{t-1}\right) \approx \sum_{i=1}^{n} p_{i} \Phi\left(\frac{\theta_{t-1}-\mu_{i}}{\sigma_{i}}\right)
$$

where $\sum_{i=1}^{n} p_{i}=1$ and $p_{i} \geq 0, \Phi(\cdot)$ denotes the cumulative probability distribution functions of standard normal distribution.

(3) The weights and distribution parameters of the fitted normal distributions can be obtained with the least residual error quadratic sum method OLS; namely,

$$
\text { OLS }=\sqrt{\frac{1}{n} \sum_{j=1}^{k}\left(g\left(\theta_{t-1}\right)-\left(\sum_{i=1}^{n} p_{i} \Phi\left(\frac{\theta_{t-1}-\mu_{i}}{\sigma_{i}}\right)\right)\right)^{2}},
$$

where $p_{i}$ is the weight. The values of unknown parameters for the fitted distributions can be possessed by the optimization computation with the rule of OLS. Furthermore, the optimized parameters must be determined to make sure that the value of OLS is the minimum.

3.3. Combinatorial Probability Recursion of BDLMs. BDLMs are applicable to the prediction of the future state parameters, which can be recursive and updated like well-known Kalman filter [15]. With Bayesian method, the combinatorial recursively updating processes $[15,18,19]$ are as follows:

(1) The a posteriori distribution at time $t-1$ : for the mean $m_{i, t-1}$ and the variance $C_{i, t-1}(i=1,2, \ldots, s)$, there is

$$
\left(\theta_{i, t-1} \mid \mathbf{D}_{i, t-1}\right) \sim N\left[m_{i, t-1}, C_{i, t-1}\right] .
$$

(2) The a priori distribution at time $t$ :

$$
\left(\theta_{i, t} \mid \mathbf{D}_{i, t-1}\right) \sim N\left[a_{i, t}, R_{i, t}\right],
$$

where $a_{i, t}=m_{i, t-1}+r, R_{i, t}=C_{i, t-1}+W_{i, t} G_{i, t}$. 
(3) One-step prediction distribution at time $t$ :

$$
\left(y_{i, t} \mid \mathbf{D}_{i, t-1}\right) \sim N\left[f_{i, t}, Q_{i, t}\right],
$$

where $f_{i, t}=E\left(y_{i, t} \mid y_{i, 1: t-1}\right)=a_{i, t}, Q_{i, t}=\operatorname{var}\left(y_{i, t} \mid y_{i, 1: t-1}\right)=$ $R_{i, t}+V_{i, t}$.

According to the definition of highest a posterior density (HPD) region [15], the predicted interval of the monitored data with a $95 \%$ confidential interval at time $t$ is

$$
\left[f_{i, t}-1.645 \sqrt{Q_{i, t}}, f_{i, t}+1.645 \sqrt{Q_{i, t}}\right],
$$

where $f_{i, t}-1.645 \sqrt{Q_{i, t}}$ is the predicted lower limit value and $f_{i, t}+1.645 \sqrt{Q_{i, t}}$ is the predicted upper limit value.

(4) The a posteriori distribution at time $t$ :

$$
\left(\theta_{i, t} \mid \mathbf{D}_{i, t}\right) \sim N\left[m_{i, t}, C_{i, t}\right]
$$

where $m_{i, t}=a_{i, t}+A_{i, t} e_{i, t} ; C_{i, t}=R_{i, t}-A_{i, t} Q_{i, t} A_{i, t}^{\prime} ; A_{i, t}=$ $R_{i, t} / Q_{i, t} ; e_{i, t}=y_{i, t}-f_{i, t}$ (one-step predicted error); $A_{i, t}$ is adaptive coefficient; and $A_{i, t}^{\prime}$ is the transpose of $A_{i, t}$.

(5) Predicted probability distribution based on the arithmetic mean at time $t$ :

$$
P_{m, t}=\sum_{i=1}^{s} \frac{1}{s} N\left[f_{i, t}, Q_{i, t}\right]=N\left[f_{m, t}, Q_{m, t}\right],
$$

where $f_{m, t}=(1 / s) \sum_{i=1}^{s} f_{i, t}, Q_{m, t}=\left(1 / s^{2}\right) \sum_{i=1}^{s} Q_{i, t}$, and $s$ is the total number of BDLMs.

(6) The combinatorial prediction probability distribution at time $t$ :

$$
P_{c, t}=\sum_{i=1}^{s} k_{i, t} N\left[f_{i, t}, Q_{i, t}\right]=N\left[f_{c, t}, Q_{c, t}\right],
$$

where $f_{c, t}=\sum_{i=1}^{s} k_{i, t} f_{i, t}, k_{i, t}=Q_{i, t}^{-1} / \sum_{i=1}^{s} Q_{i, t}^{-1}, i=1,2, \ldots, s$, and $\sum_{i=1}^{s} k_{i, t}=1, Q_{c, t}^{-1}=\sum_{i=1}^{s} Q_{i, t}^{-1} \cdot Q_{i, t}^{-1}$ is the predicted precision of the $i$ th model (the reciprocal of the one-step predicted variance) and $Q_{c, t}^{-1}$ is the predicted precision of the combinatorial prediction model.

(7) Comparison of the predicted precisions between combinatorial prediction model and the prediction model based on the arithmetic means:

$$
Q_{c, t}^{-1} \geq Q_{m, t}^{-1},
$$

where $Q_{c, t}^{-1}=\sum_{i=1}^{s} Q_{i, t}^{-1}, Q_{m, t}^{-1}=s^{2} / \sum_{i=1}^{s} Q_{i, t}$.

3.4. Model Monitoring of BDLMs. Model monitoring has three purposes. The first is to identify where model prediction function declines and which form the model fault occurred in. The second is to cope with the faults and to monitor and update the model. The third is to improve the accuracy of future prediction.

In this paper, the main idea of model monitoring mechanism is to use one or more alternative models to compare and evaluate model performance.
According to the research [17], model monitoring is achieved through Bayesian factors under the normal assumption. The main idea is firstly to build an alternative model and then to combine an existing probability model for constructing the formula of Bayesian factors.

In this paper, the adopted probability distribution density function of the alternative model and one-step prediction model are, respectively, (31) and (32) as follows:

$$
\begin{aligned}
& p_{1}=\left(2 \pi k^{2}\right)^{-0.5} \exp \left(-\frac{0.5 e_{t e}^{2}}{k^{2}}\right), \\
& p_{0}=(2 \pi)^{-0.5} \exp \left(-0.5 e_{t e}^{2}\right),
\end{aligned}
$$

where $e_{t e}=\left(y_{t}-f_{t}\right) / \sqrt{Q_{t}}$ is the standard prediction error and $k$ is the standard deviation of $e_{t e}$.

The Bayesian factor for $p_{0}(\cdot)$ versus $p_{1}(\cdot)$ based on the observed value of $y_{t}$ is defined as

$$
B(t)=\frac{p_{0}\left(y_{t} \mid \mathbf{D}_{t-1}\right)}{p_{1}\left(y_{t} \mid \mathbf{D}_{t-1}\right)},
$$

where $p_{0}\left(y_{t} \mid \mathbf{D}_{t-1}\right)$ is the one-step predictive probability density function of monitored extreme stress data; $p_{1}\left(y_{t} \mid\right.$ $\left.\mathbf{D}_{t-1}\right)$ is probability density function of the alternative model, namely, the routine or standard probability density function; $B(t)$ is the Bayesian factor for $p_{0}\left(y_{t} \mid \mathbf{D}_{t-1}\right)$ versus $p_{1}\left(y_{t} \mid\right.$ $\mathbf{D}_{t-1}$ ) based on the observed value of $y_{t}$.

Further, according to (31)-(33), the Bayesian factors can be obtained as follows:

$$
B(t)=k \exp \left\{-0.5 e_{t e}^{2}\left(1-k^{-2}\right)\right\},
$$

where $e_{t e}=\left(y_{t}-f_{t}\right) / \sqrt{Q_{t}}$ is the standard prediction error and $k$ is the standard deviation of $e_{t e}$.

For integers $N=1, \ldots, t$, the Bayesian factor for $p_{0}(\cdot)$ versus $p_{1}(\cdot)$ based on the sequence of $N$ consecutive observations $y_{t}, y_{t-1}, \ldots, y_{t-N+1}$ is built as (35); namely, the built formula $B_{t}(N)$ of the cumulative Bayesian factor is

$$
\begin{aligned}
B_{t}(N) & =\prod_{r=t-N+1}^{t} B_{r} \\
& =\frac{p_{0}\left(y_{t}, y_{t-1}, \ldots, y_{t-N+1} \mid \mathbf{D}_{t-N}\right)}{p_{1}\left(y_{t}, y_{t-1}, \ldots, y_{t-N+1} \mid \mathbf{D}_{t-N}\right)}
\end{aligned}
$$

where $B_{t}(N)$ is the cumulative Bayesian factor, which measures the evidence provided by the recent (up to and including time $t) N$ consecutive observations $y_{t}, y_{t-1}, \ldots, y_{t-N+1}$.

With (34), the changing curves of Bayesian factors with $k$ are shown in Figure 2. In this paper, according to the engineering experience, the adopted monitoring criteria [15, $16,21]$ are as follows: if $k=3$ and $B(t)<0.15$, then the corresponding inspection/monitored data is abnormal, which need to be removed; otherwise, the inspection/monitored data is normal. 


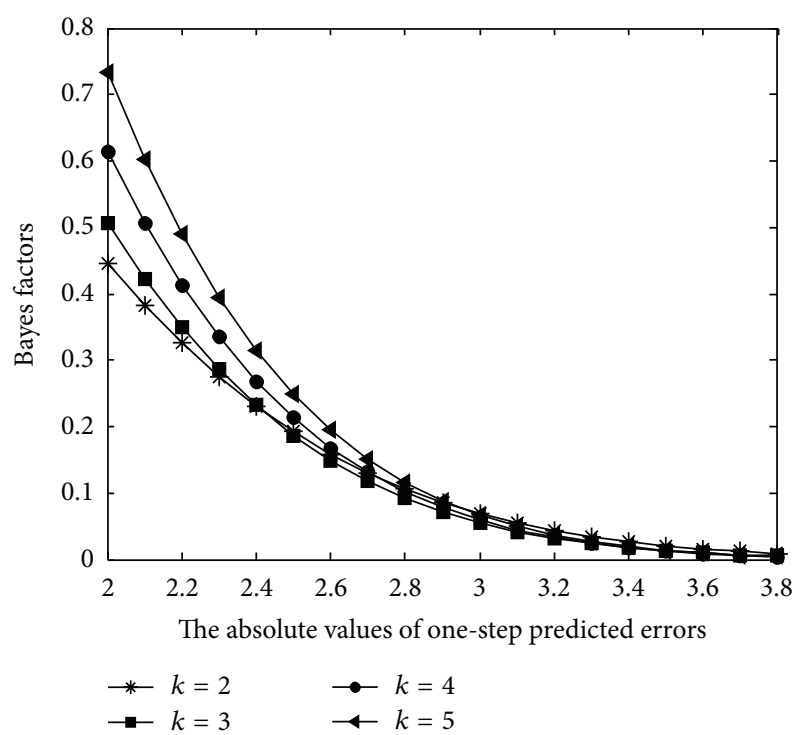

FIgURE 2: Curves of Bayes factors versus one-step predicted errors.

After removing the abnormal data, in the changing curves of cumulative Bayesian factors, the prediction precision of the Bayesian dynamic model can be shown. Namely, if the cumulative Bayesian factor is bigger, the prediction precision of the Bayesian dynamic model is better. The uncertainty of the Bayesian dynamic models is smaller.

\section{Reliability Prediction Based on Combinatorial BDLMs}

4.1. First-Order Second-Moment (FOSM) Method. In this paper, the FOSM method [2,3] is adopted to predict the reliability indices of bridge structures. Namely, only the mean and variance of predicted data are taken into account.

Suppose there are random variables $R$ (generalized resistance) and $S$ (generalized load effects including dead load effect and live load effect) which are internally independent and mutually independent; the mean value and standard variance of which are, respectively, as follows: $\mu_{R}, \sigma_{R}$; $\mu_{S}, \sigma_{S}$.

With FOSM method, the formula of the reliability indices can be obtained with

$$
\beta_{t}=\frac{\mu_{R}-\mu_{S}}{\sqrt{\sigma_{R}^{2}+\sigma_{S}^{2}}} .
$$

4.2. Prediction Formula of Reliability Indices Based on FOSM. In this paper, a five-span continuous steel plate girder bridge is taken as an example. The total length of the bridge is $188.81 \mathrm{~m}$. The explicit details about the aim and results of the monitoring program for the whole bridge are given in [11]. The extreme stress data at the beam bottom in the middle part of the second lateral span from the whole bridge is monitored. As far as the actual engineering is concerned, the computation accuracy of the reliability method (firstorder second-moment (FOSM)) [3] adopted in this paper is sufficient. Namely, only the mean value and second-moment value about the variable are used. The limit state function of the beam from the second lateral span is

$$
g(R, S, C, M)=R-S-C-\gamma_{M} M
$$

where $R$ is steel yield strength, $S$ is the stress caused by the dead weight of steel, $C$ is the stress caused by the dead weight of concrete, $M$ is the monitored extreme stresses predicted with the combinatorial BDLMs, and $\gamma_{M}$ is a factor assigned to the data provided by the sensors.

The reliability index $\beta_{p}$ (first-order) is

$$
\beta_{p}=\frac{\mu_{R}-\mu_{S}-\mu_{C}-\gamma_{M} \times \mu_{M}}{\sqrt{\sigma_{R}^{2}+\sigma_{S}^{2}+\sigma_{C}^{2}+\left(\gamma_{M} \times \sigma_{M}\right)^{2}}},
$$

where $\mu_{M}, \sigma_{M}$ are mean and standard deviation of $M ; \mu_{R}$, $\sigma_{R}$ are mean and standard deviation of $R ; \mu_{S}, \sigma_{S}$ are mean and standard deviation of $S ; \mu_{C}, \sigma_{C}$ are mean standard deviation of $C ; \gamma_{M}$ is a factor assigned to the data provided by the sensors.

For the real-time monitored reliability indices, the monitored data is one by one, so $\sigma_{M}=0$, while for the reliability indices predicted with the combinatorial BDLMs in this paper, due to the randomness and the uncertainty of the monitored data, $\sigma_{M} \neq 0$.

\section{Application to an Existing Bridge}

The I-39 Northbound Bridge, which was described in Section 4.2, was built in 1961; it is a five-span continuous steel plate girder bridge. The extreme stresses at the beam bottom in the middle part of the second lateral span from the whole bridge are monitored for eighty-three days; the monitored data displayed the variability of the stresses caused by traffic, temperature, shrinkage, creep, and structural changes. The stresses from the dead weight of the steel structure and the concrete deck are not included in the measured data. And the day-by-day monitored extreme stress data are shown in Table 1 and Figure 3.

In this existing example, the state equation, obtained with (4)-(8), is adopted to build the BDLMs; namely, 1-order polynomial function of monitored data is

$$
m_{t}=-0.0321 t+25.71 \text {, }
$$

where $m_{t}$ is approximately state value of health monitored data at time $t$.

For obtaining the distribution parameters of initial information, the monitored extreme data of the 83 days is smoothly processed, and then the initial information of monitored data is approximately obtained, which is shown in Figure 4.

Through Kolmogorov-Smirnov (K-S) test for the initial information, the initial a priori PDF is lognormal PDF or normal PDF shown in Figure 5 and (42). 
TABLE 1: Real-time monitored extreme stresses.

\begin{tabular}{|c|c|c|c|c|c|c|c|}
\hline Time $t$ (day) & Stress $\sigma(\mathrm{MPa})$ & Time $t$ (day) & Stress $\sigma(\mathrm{MPa})$ & Time $t$ (day) & Stress $\sigma(\mathrm{MPa})$ & Time $t$ (day) & Stress $\sigma(\mathrm{MPa})$ \\
\hline 1 & 25.23 & 22 & 21.22 & 43 & 24.17 & 64 & 25.15 \\
\hline 2 & 21.67 & 23 & 22.02 & 44 & 23.72 & 65 & 24.64 \\
\hline 3 & 19.53 & 24 & 34.80 & 45 & 26.85 & 66 & 23.18 \\
\hline 4 & 20.50 & 25 & 30.51 & 46 & 30.32 & 67 & 21.94 \\
\hline 5 & 24.44 & 26 & 21.57 & 47 & 31.93 & 68 & 18.82 \\
\hline 6 & 22.66 & 27 & 31.67 & 48 & 25.06 & 69 & 22.66 \\
\hline 7 & 25.95 & 28 & 29.16 & 49 & 23.01 & 70 & 21.57 \\
\hline 8 & 32.65 & 29 & 21.67 & 50 & 22.02 & 71 & 29.16 \\
\hline 9 & 39.26 & 30 & 23.99 & 51 & 33.90 & 72 & 21.57 \\
\hline 10 & 21.40 & 31 & 21.05 & 52 & 18.10 & 73 & 32.92 \\
\hline 11 & 31.48 & 32 & 29.35 & 53 & 25.24 & 74 & 21.94 \\
\hline 12 & 30.06 & 33 & 22.66 & 54 & 25.77 & 75 & 21.14 \\
\hline 13 & 20.60 & 34 & 24.61 & 55 & 17.11 & 76 & 20.41 \\
\hline 14 & 22.56 & 35 & 25.77 & 56 & 23.72 & 77 & 16.76 \\
\hline 15 & 23.54 & 36 & 28.54 & 57 & 12.65 & 78 & 22.38 \\
\hline 16 & 16.94 & 37 & 22.83 & 58 & 24.89 & 79 & 27.21 \\
\hline 17 & 29.16 & 38 & 21.05 & 59 & 27.56 & 80 & 19.98 \\
\hline 18 & 22.47 & 39 & 24.44 & 60 & 25.86 & 81 & 18.82 \\
\hline 19 & 23.37 & 40 & 28.80 & 61 & 24.61 & 82 & 29.44 \\
\hline 20 & 28.99 & 41 & 20.24 & 62 & 22.11 & 83 & 20.41 \\
\hline 21 & 30.15 & 42 & 29.97 & 63 & 21.22 & & \\
\hline
\end{tabular}

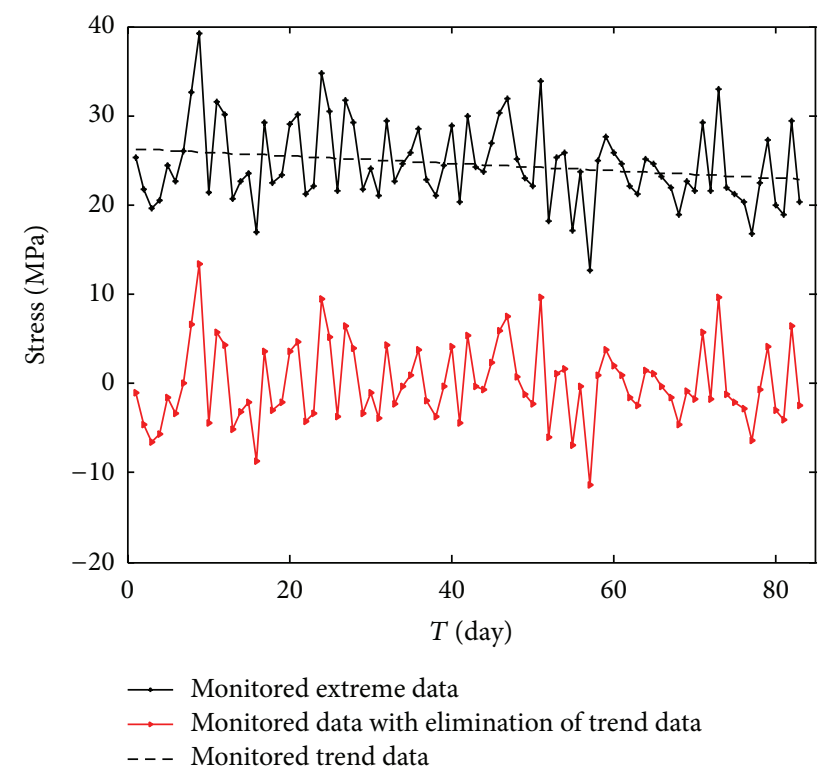

FIGURE 3: Curves of monitored extreme stresses.

5.1. BDLMs Based on the Monitored Data. Based on the monitored data, with (4)-(8) and (9)-(11), the built BDLMs are as follows:

observation equation:

$$
y_{t}=m_{t}+v_{t}, \quad v_{t} \sim N[0, V]
$$

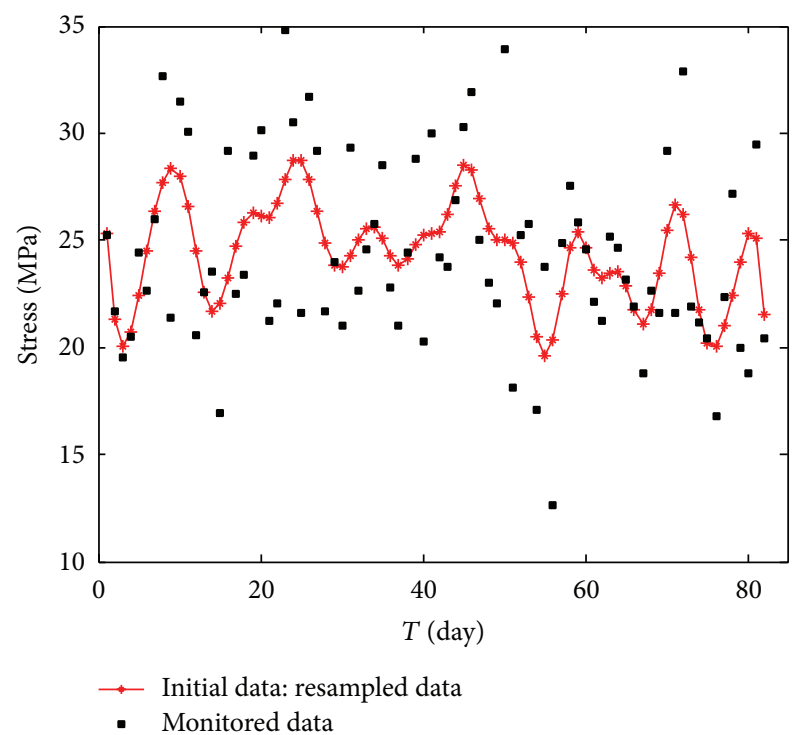

FIGURE 4: Curves of initial information and the monitored extreme stress data.

state equation:

$$
m_{t}=m_{t-1}-0.0321+\omega_{t}, \quad \omega_{t} \sim N\left[0, W_{t}\right] ;
$$

initial information:

$$
\begin{aligned}
m_{t-1} & \mid \mathbf{D}_{t-1} \\
& \sim N\left[24.5052,4.6635^{2}\right] \text { or } \operatorname{LN}\left[3.1811,0.1886^{2}\right],
\end{aligned}
$$




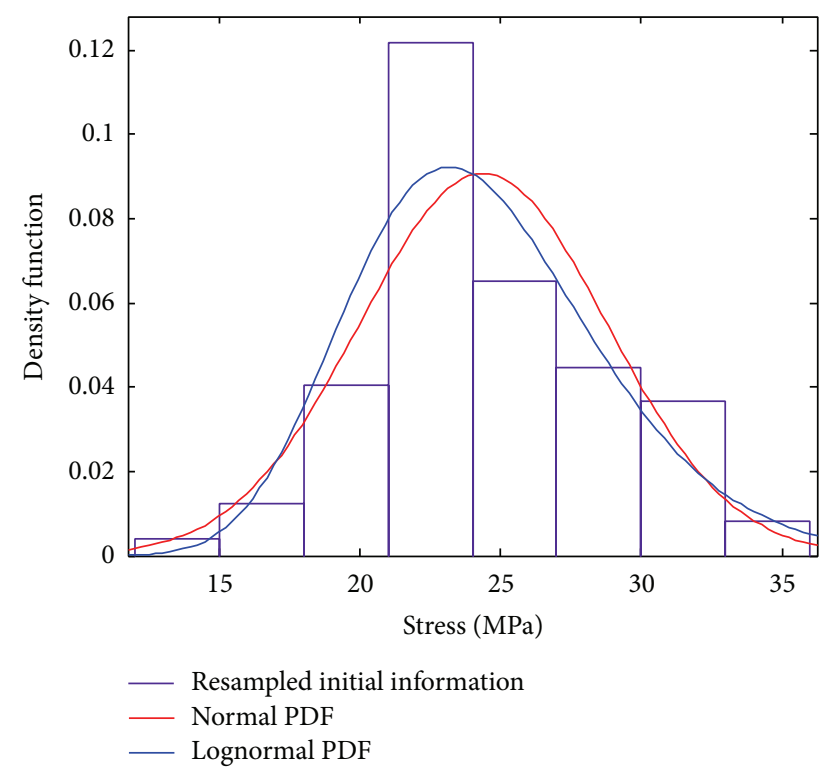

FIGURE 5: PDF curves fitted with the initial monitored extreme stress data (PDF: probability density function).

where $y_{t}$ is the monitored extreme data at time $t ; m_{t}$ is the state value of the monitored extreme data at time $t ; v_{t}$ is monitored error; $\omega_{t-1}$ is state error $V=21.75$ which can be approximately obtained with monitored extreme data; and $\delta=0.98, W_{t}=-C_{t-1}+C_{t-1} / \delta$ according to the actual engineering experience of the authors. $C_{t-1}$ can be obtained with (18)-(19) and (42). N[·] means normal probability distribution and $L N[\cdot]$ means lognormal probability distribution.

Equation (42) shows that the initial information follows normal distribution or lognormal distribution. So the following four cases are discussed to predict the monitored extreme data.

Case 1. Initial information follows normal distribution, and then the BDLMs are built to predict the monitored extreme data.

Case 2. Initial information follows lognormal distribution; firstly the lognormal distribution must be transformed into a quasinormal distribution [21, 22] with (18)-(19); and then the BDLMs are built based on the quasinormal distribution to predict the monitored extreme data.

Case 3. The arithmetic mean of the one-step predicted mean values, respectively, obtained with Cases 1 and 2 is considered as the predicted extreme data of the third case.

Case 4. The fourth case is to build combinatorial BDLMs with BDLMs obtained with Cases 1 and 2; the modeling processes of combinatorial BDLMs are described in Sections 3.2 and 3.3.

In this paper, the Bayesian factors are adopted to seek the abnormal data, and the monitored results are shown in Figures 6 and 7, from which it can be known that the data

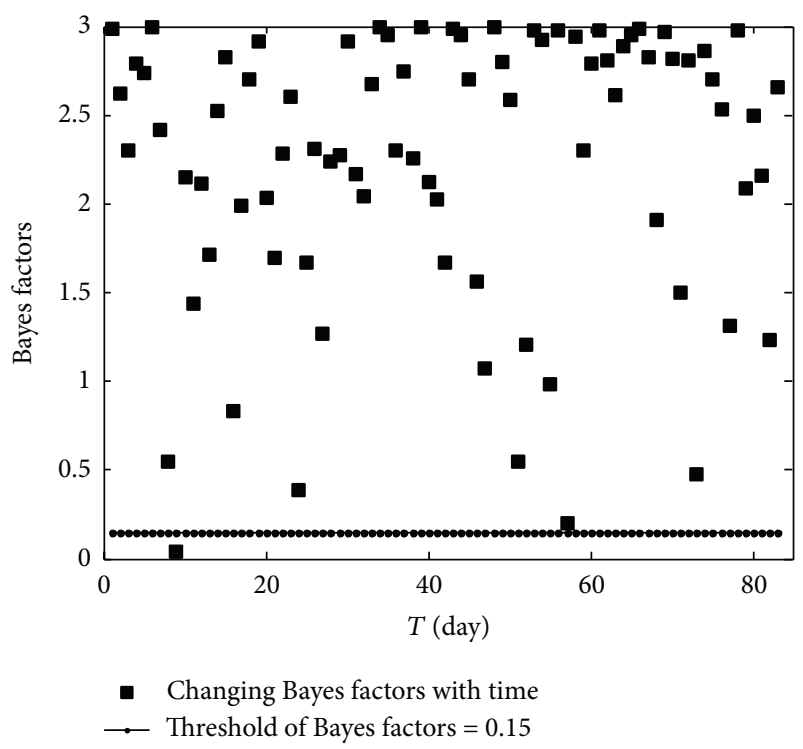

Figure 6: Curves of time-dependent Bayes factors (the data of the 9 th day is abnormal).

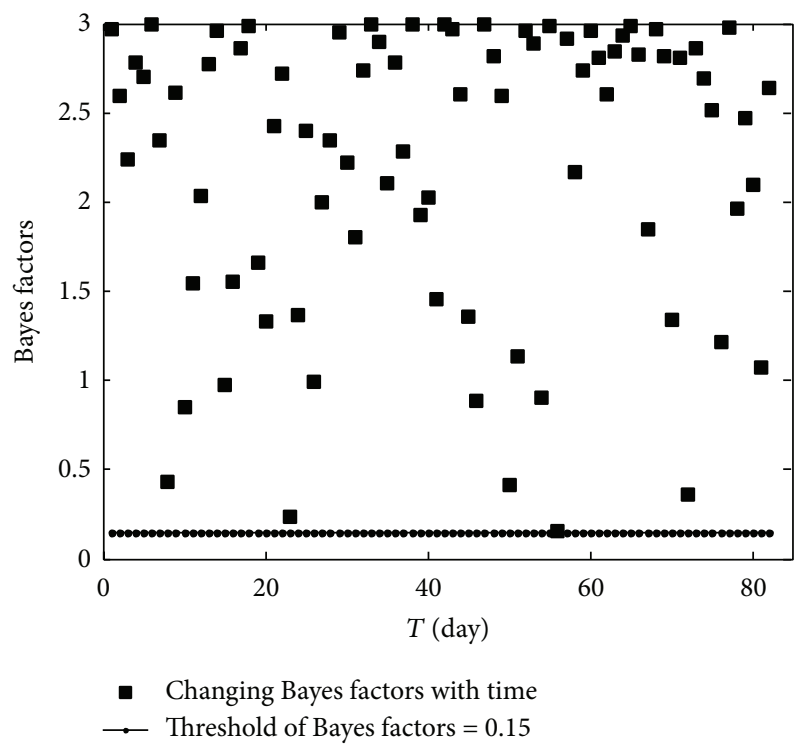

FIgURE 7: Curves of time-dependent Bayes factors after eliminating the abnormal extreme data (the data of the 9th day is deleted).

of the 9th day is abnormal. From Table 1, it can be seen that the data of the 9th day is the biggest, so it may be abnormal. After removal of the abnormal data, the changing cumulative Bayesian factors shown in Figure 8 reflect that the prediction precision of BDLMs is better and better.

The predicted extreme stresses and prediction precision (the reciprocal of predicted variances) of the above four cases are, respectively, shown in Figures 9-14.

From Figures 9-13, it can be noticed that the predicted data and the predicted ranges of the four cases all fit the changing rules of monitored extreme data, but as far as the prediction precision shown in Figure 14 is concerned, 


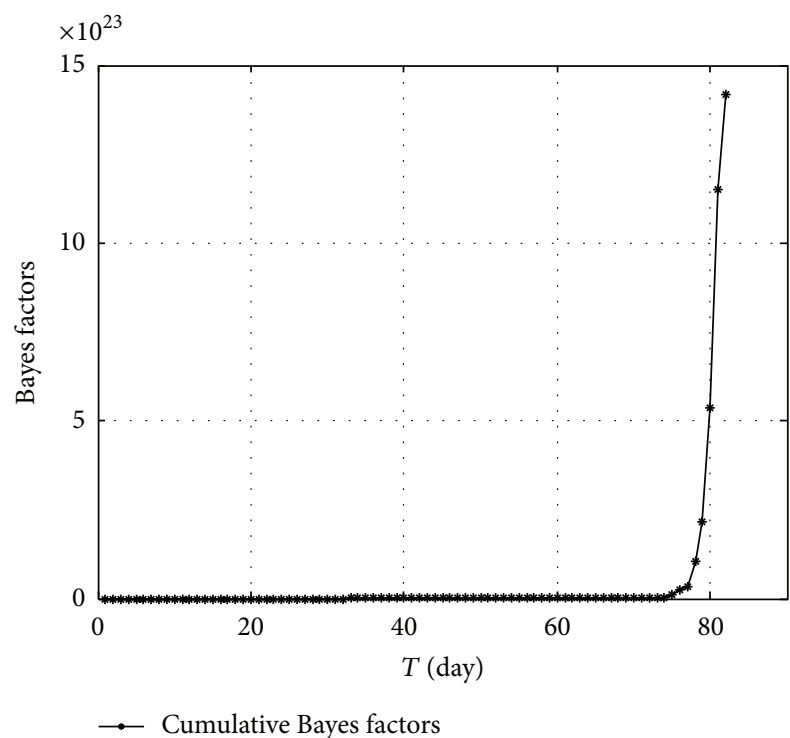

FIGURE 8: Curves of cumulative Bayesian factors.

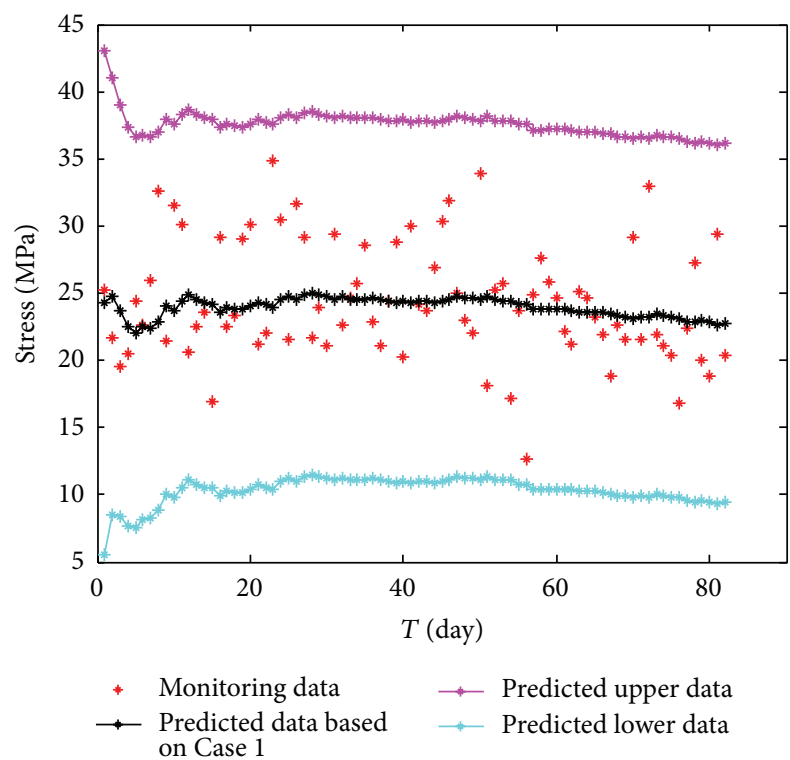

FIGURE 9: Predicted curves of extreme data when initial information follows normal probability distribution (Case 1).

the prediction precision of combinatorial model is the best. So the combinatorial prediction model of monitored extreme data is adopted to predict the structural reliability indices.

\subsection{Reliability Prediction Based on the Combinatorial BDLMs.} In Figure 14, it can be observed that prediction precision of the combinatorial model is the best. So the combinatorial model of the extreme data is adopted to predict the structural reliability indices with (38) and (43). The predicted results, which are shown in Figure 15, can approximately show the changing trends and changing ranges of monitored reliability indices. The design specifications $\mu_{R}=380 \mathrm{MPa}$ and $\sigma_{R}=380 \times 0.07=26.6 \mathrm{MPa}, \mu_{S}=116.3 \mathrm{MPa}$ and $\sigma_{S}=116.3 \times 0.04=4.65 \mathrm{MPa}$, and $\mu_{C}=108.8 \mathrm{MPa}$ and

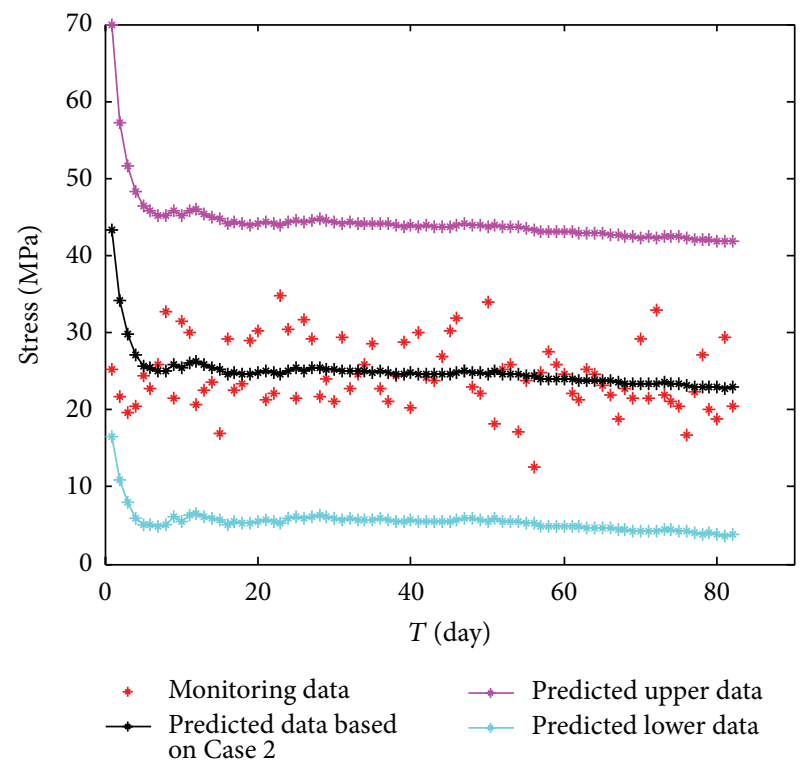

FIGURE 10: Predicted curves of extreme data when initial information follows lognormal probability distribution (Case 2).

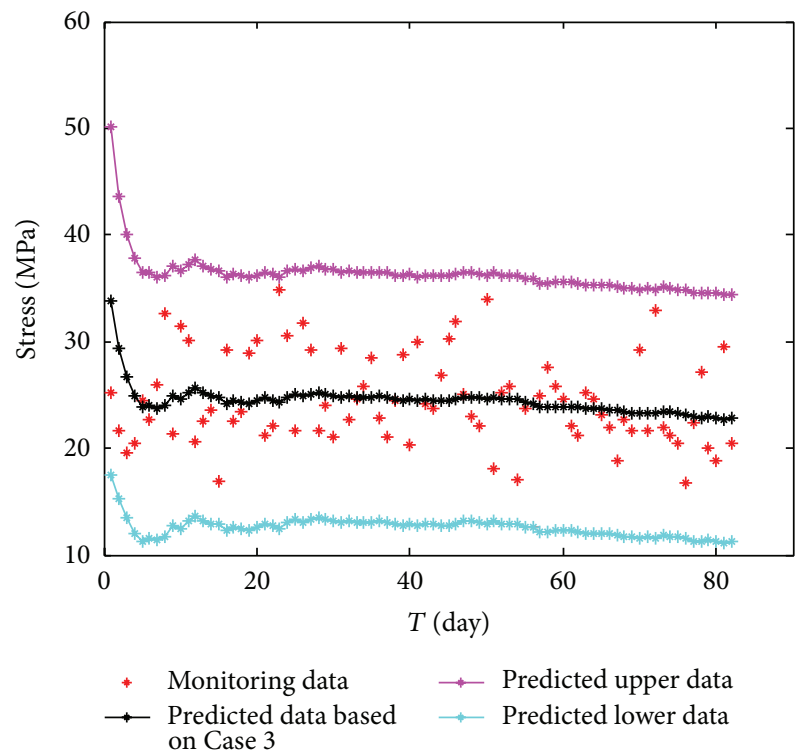

FIGURE 11: Predicted curves of extreme data based on the arithmetic mean of the two distributions (Case 3).

$\sigma_{\mathrm{C}}=108.8 \times 0.04=4.35 \mathrm{MPa}$ of the stresses of the structural dead weight yield to

$$
\begin{aligned}
\beta_{p} & =\frac{\mu_{R}-\mu_{S}-\mu_{C}-\gamma_{M} \times \mu_{M}}{\sqrt{\sigma_{R}^{2}+\sigma_{S}^{2}+\sigma_{C}^{2}+\left(\gamma_{M} \times \sigma_{M}\right)^{2}}} \\
& =\frac{380-116.3-108.8-1.15 \mu_{M}}{\sqrt{26.6^{2}+4.65^{2}+4.35^{2}+\left(1.15 \times \sigma_{M}\right)^{2}}} \\
& =\frac{155-1.15 \times \mu_{M}}{\sqrt{27.351^{2}+\left(1.15 \times \sigma_{M}\right)^{2}}}
\end{aligned}
$$




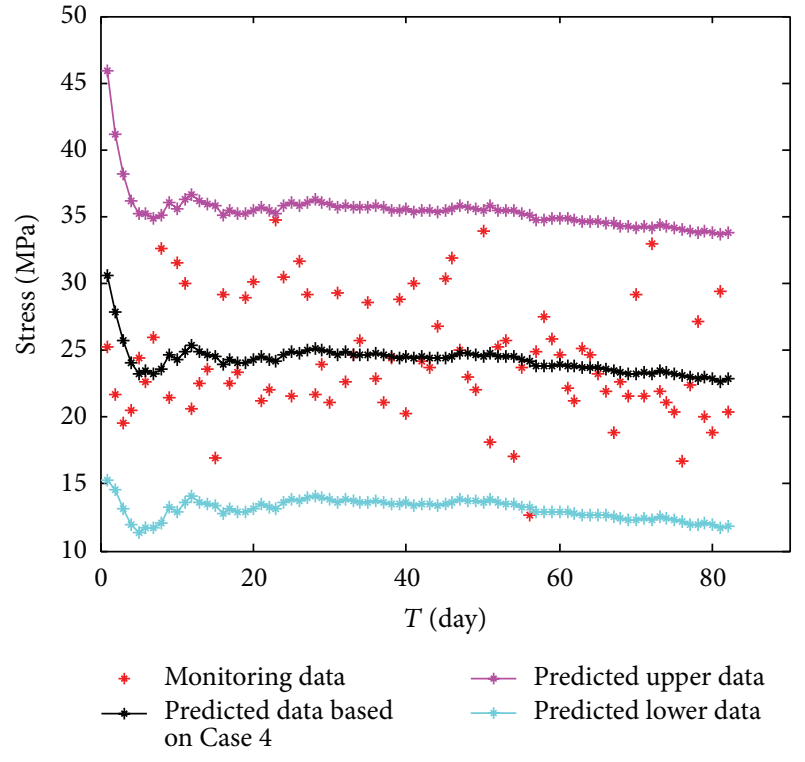

FIgURE 12: Predicted curves of extreme data based on the combinatorial model (Case 4).

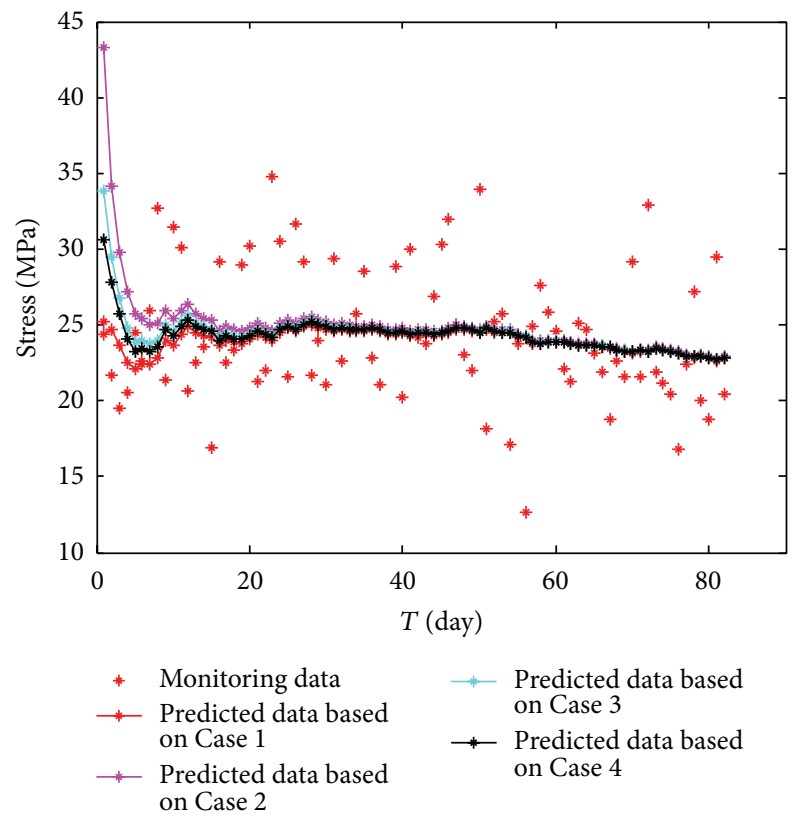

FIGURE 13: Comparison among predicted data of the four cases.

assigned to bottom of the girder in the middle of the second lateral span which is shown in [19], where $\mu_{M}, \sigma_{M}$ are mean and standard deviation of the monitored extreme stresses predicted with the combinatorial BDLMs.

\section{Conclusions}

In this paper, based on the everyday monitored extreme stresses of bridge, the structural reliability indices are

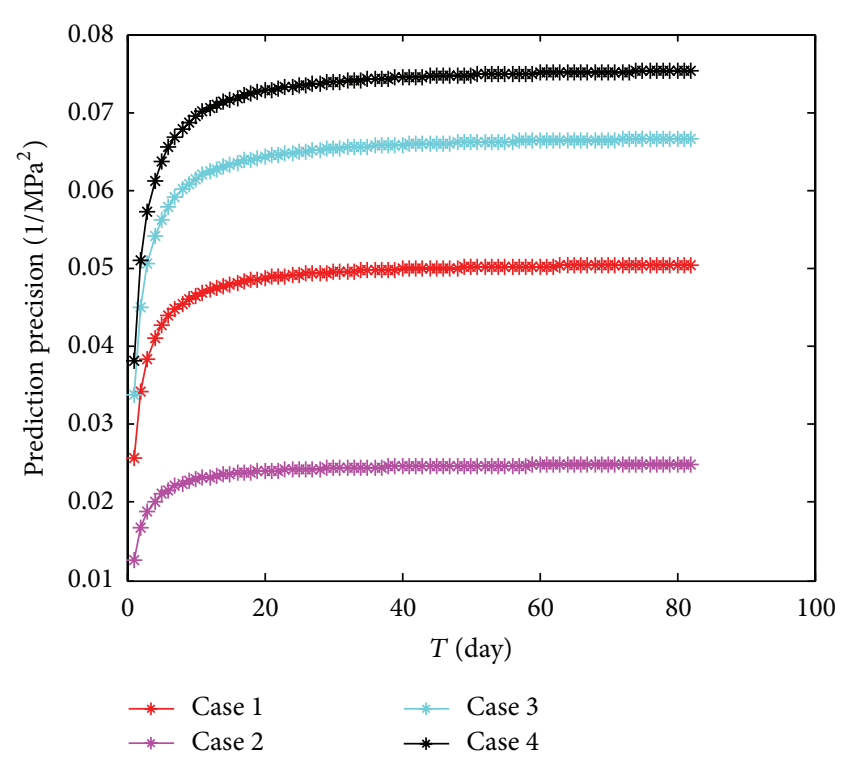

FIGURE 14: Prediction precision comparisons among the predicted stresses of the four cases.

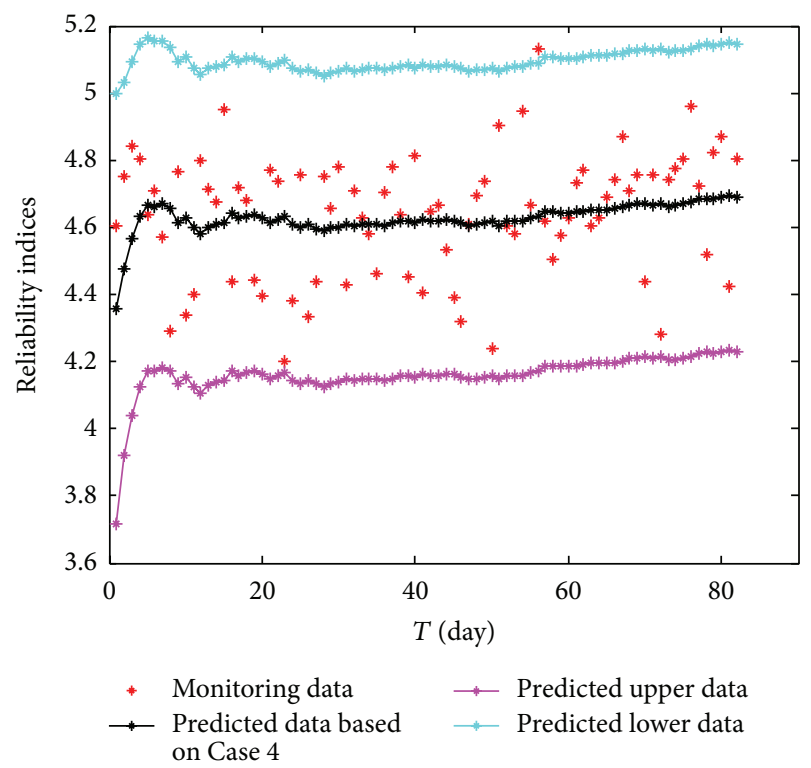

FIGURE 15: Curves of reliability indices based on the combinatorial model of monitored extreme data.

predicted with combinatorial BDLMs and FOSM method. And the following conclusions can be reached:

(1) The BDLMs, which are used to seek the abnormal data of the mass monitoring information, are obtained with 1-order polynomial function based on the past information.

(2) The monitored extreme stresses-based combinatorial BDLMs are firstly built. The predicted extreme stresses and the predicted ranges of the above four 
cases are almost the same, but as far as the prediction precision is concerned, the combinatorial BDLMs have the best prediction precision.

(3) Based on the combinatorial BDLMs of monitored extreme stresses, structural reliability indices are predicted. Compared with deterministic monitored extreme stresses-based reliability indices, this paper considered the randomness and uncertainty of monitored data, so the predicted reliability indices are smaller. But the predicted smaller reliability indices may better reflect the actual state of the bridge. Thus, the smaller reliability indices may be more reasonably used to assess the structural safety and serviceability.

In this paper, the proposed reliability prediction method is easy and may be widely used in the structural health monitoring. BDLMs are possible to include subjective judgments with the observed data in order to obtain a more informed and accurate prediction. The numerical applications presented, using the monitored extreme data of an existing bridge, illustrate the application and feasibility of the proposed approaches and concepts.

\section{Conflict of Interests}

The authors declare that there is no conflict of interests regarding the publication of this paper.

\section{Acknowledgment}

This work was supported by the Fundamental Research Funds for the Central Universities (lzujbky-2015-300, lzujbky-2015-301).

\section{References}

[1] D. Straub and M. H. Faber, "Computational aspects of riskbased inspection planning," Computer-Aided Civil and Infrastructure Engineering, vol. 21, no. 3, pp. 179-192, 2006.

[2] A. H.-S. Ang and W. H. Tang, Probability Concepts in Engineering Planning and Design, vol. 2, John Wiley \& Sons, New York, NY, USA, 1984.

[3] R. E. Melchers, Structural Reliability, Analysis and Prediction, Ellis Horwood, Chichester, UK, 1987.

[4] A. Q. Li and C. Q. Miu, "Health monitoring system for the Runyang Yangtse River Bridge," Journal of Southeast University, vol. 33, no. 5, pp. 544-548, 2003 (Chinese).

[5] T. H. T. Chan, L. Yu, H. Y. Tam et al., "Fiber Bragg grating sensors for structural health monitoring of Tsing Ma bridge: background and experimental observation," Engineering Structures, vol. 28, no. 5, pp. 648-659, 2006.

[6] H. Li, W. Zhou, J. Ou, and Y. Yang, "A study on system integration technique of intelligent monitoring systems for soundness of long-span bridges," China Civil Engineering Journal, vol. 39, no. 2, pp. 46-52, 2006 (Chinese).

[7] I. Hodgson, Personal discussion for the acquisition of the real data from the monitoring of the I-39 Northbound Bridge over the Wisconsin River. Ian Hodgson, Senior Research Engineer. Department of Civil and Environmental Engineering, ATLSS Center, Lehigh University, Bethlehem, PA, USA, February 12, 2007.
[8] Q. Qin, "Health monitoring of long-span bridges," China Journal of Highway and Transport, vol. 13, no. 2, pp. 37-42, 2000 (Chinese).

[9] S. L. Li, Approaches of Condition Assessment and Damage Alarming of Bridges based on Structural Health Monitoring, School of Civil Engineering, Harbin Institute of Technology, Harbin, China, 2009 (Chinese).

[10] T. Guo, A. Q. Li, and C. D. Bian, "Condition assessment of long span bridges using Monte Carlo numerical simulation," Journal of Highway and Transportation Research and Development, vol. 22, no. 8, pp. 26-30, 2005 (Chinese).

[11] H. N. Mahmoud, R. J. Connor, and C. A. Bowman, "Results of the fatigue evaluation and field monitoring of the I-39 Northbound Bridge over the Wisconsin River," ATLSS Report 05-04, Lehigh University, Bethlehem, Pa, USA, 2005.

[12] X. X. Li, Z. X. Wang, T. L. Wang, and X. D. Mei, "Assessment of dynamic reliability of existing bridges having regard to time variations of target reliability indices," Bridge Construction, supplement 1, pp. 132-134, 2007 (Chinese).

[13] Y. X. Yang, Time-Dependent Reliability Assessment and Life Prediction of Concrete Continuous Beam Bridge, School of Civil Engineering, Harbin Institute of Technology, Harbin, China, 2007 (Chinese).

[14] H. Dai, H. Zhang, W. Wang, and G. Xue, "Structural reliability assessment by local approximation of limit state functions using adaptive markov chain simulation and support vector regression," Computer-Aided Civil and Infrastructure Engineering, vol. 27, no. 9, pp. 676-686, 2012.

[15] M. West and J. Harrison, Bayesian Forecasting and Dynamic Models, Springer, New York, NY, USA, 2nd edition, 1997.

[16] J. Wang and X. L. Liu, "Evaluation and bayesian dynamic prediction of deterioration of structural performance," Structure and Infrastructure Engineering: Maintenance, Management, Life-Cycle Design and Performance, vol. 6, no. 6, pp. 663-674, 2010.

[17] X. L. Zhang, F. S. Liu, and C. J. Zhang, Bayesian Dynamic model and Forecasting, Shandong Science \& Technology Press, Jinan, China, 1992 (Chinese).

[18] G. Petris, S. Petrone, and P. Campagnoli, Dynamic Linear Models with R, Use R, Springer, New York, NY, USA, 2009.

[19] X.-P. Fan and D.-G. Lü, "Real-time reliability forecast of bridge structures based on multiple BDLMs," Journal of South China University of Technology, vol. 41, no. 3, pp. 70-75, 2013 (Chinese).

[20] J. F. Jiang, F. S. Liu, and X. L. Zhang, "Combinatorial forecast of bayesian dynamic linear model," Journal of Shandong University of Science and Technology, vol. 21, no. 3, pp. 16-17, 2002 (Chinese).

[21] D. G. Lu and X. P. Fan, "Bayesian forecasting of structural bending capacity of aging bridges based on dynamic linear model. Life-cycle and sustainability of civil infrastructure systems," in Proceedings of the 3rd International Symposium on Life-Cycle Civil Engineering (IALCCE '12), pp. 268-274, Vienna, Austria, October 2012.

[22] J. Wang, Performance Prediction and Reliability Management of Concrete Structures Based on Information Updating, School of Civil Engineering, Tsinghua University, Beijing, China, 2006 (Chinese). 


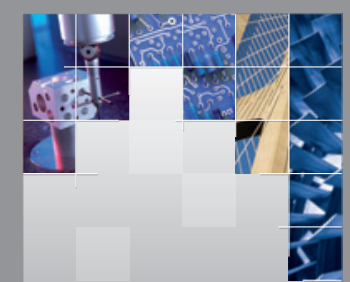

\section{Enfincering}
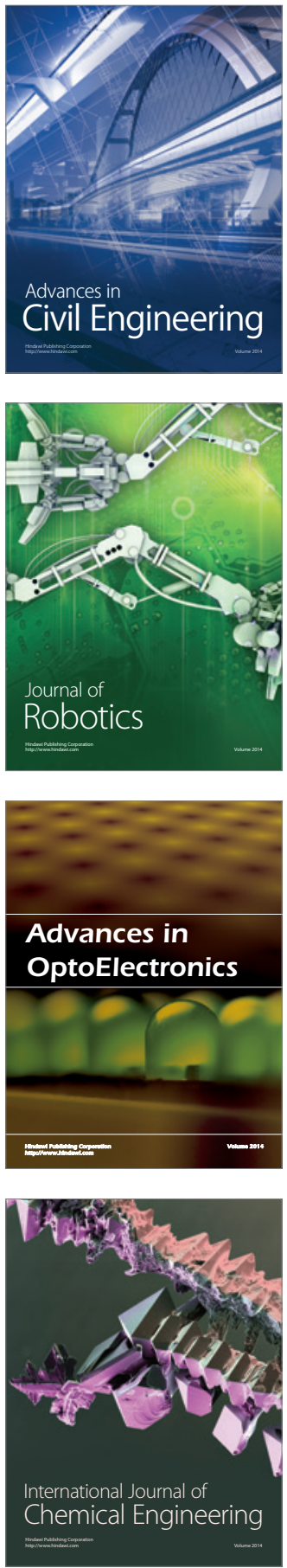

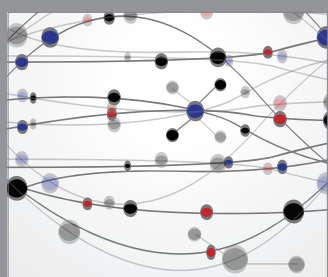

The Scientific World Journal

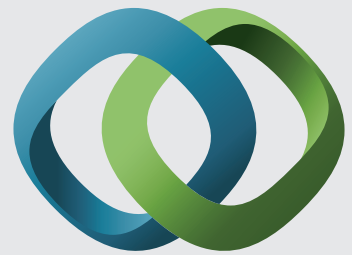

\section{Hindawi}

Submit your manuscripts at

http://www.hindawi.com
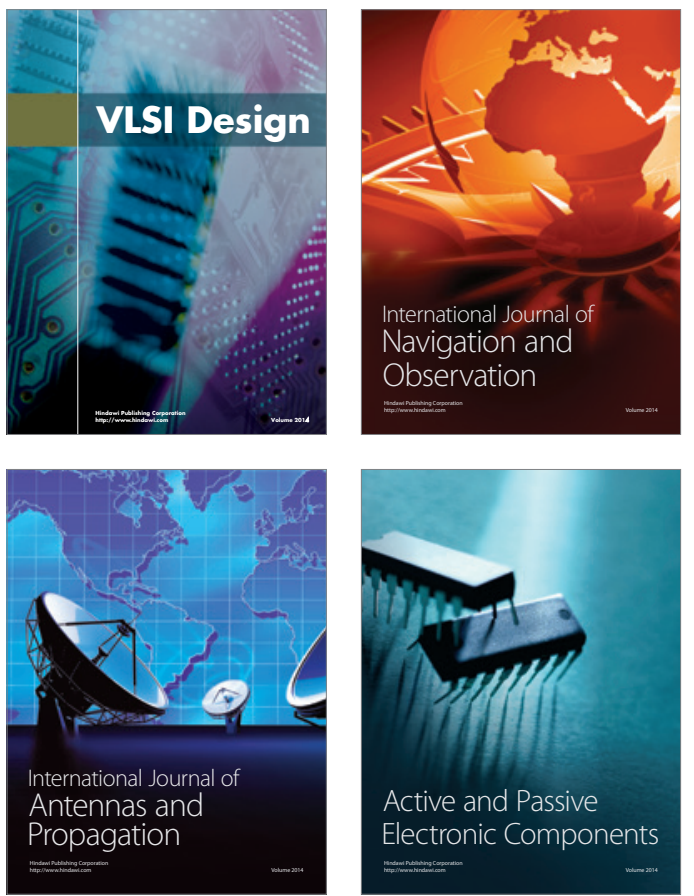
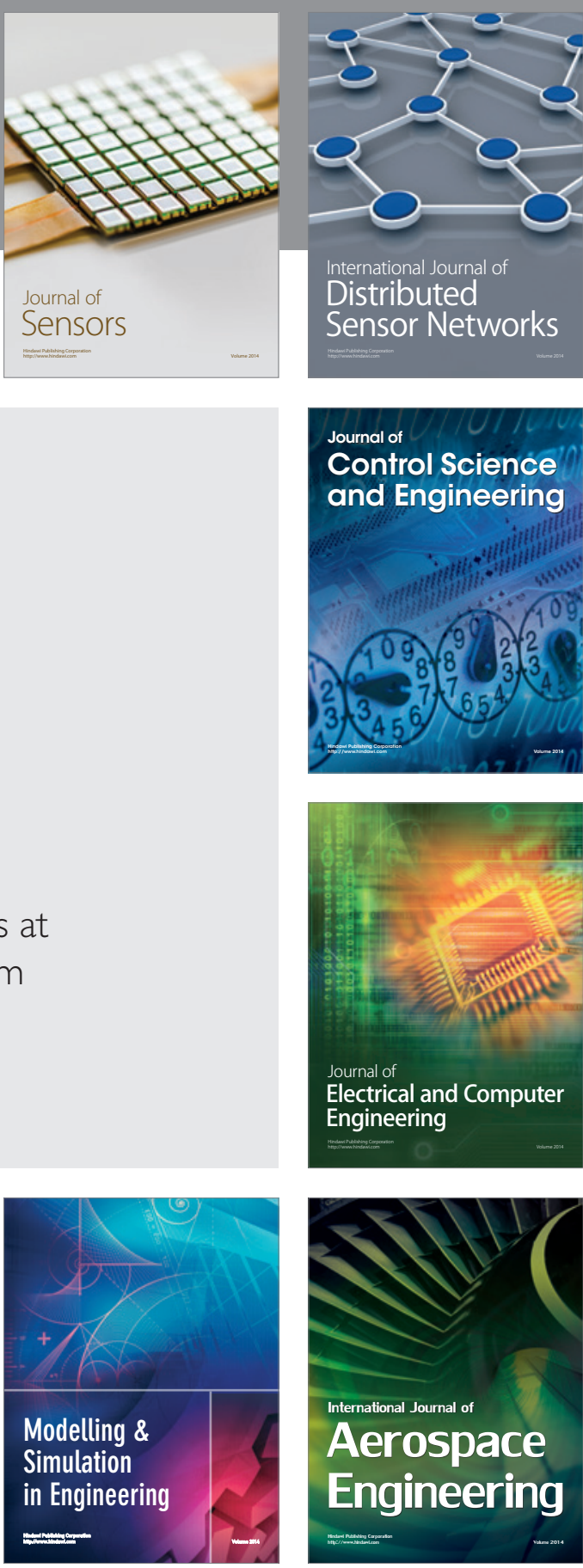

International Journal of

Distributed

Sensor Networks

Journal of

Control Science

and Engineering
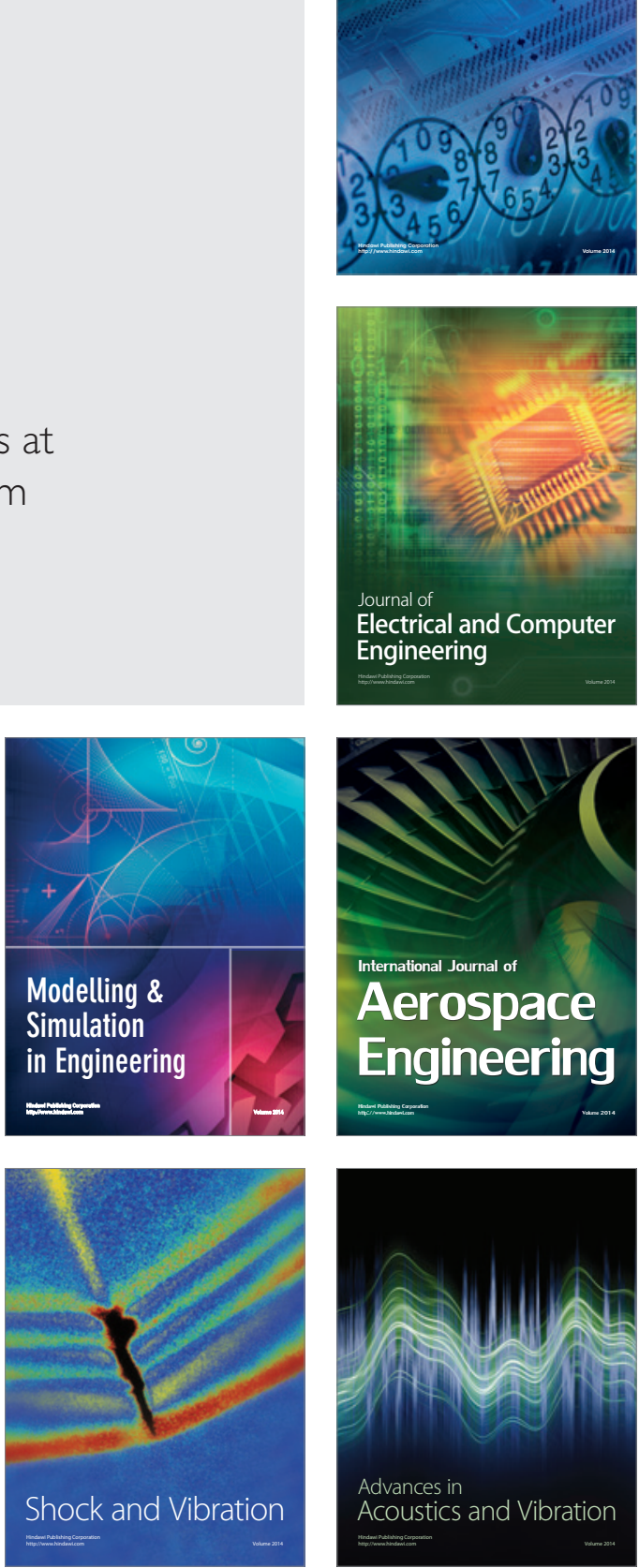